ANNALES

POLONICI MATHEMATICI

$85.2(2005)$

\title{
Period function's convexity for Hamiltonian centers with separable variables
}

\author{
by M. SABAtini (Trento)
}

\begin{abstract}
A convexity theorem for the period function $T$ of Hamiltonian systems with separable variables is proved. We are interested in systems with non-monotone $T$. This result is applied to proving the uniqueness of critical orbits for second order ODE's.
\end{abstract}

1. Introduction. Let us consider a planar Hamiltonian system with separated variables,

$$
x^{\prime}=F^{\prime}(y), \quad y^{\prime}=-G^{\prime}(x),
$$

defined on an open connected set $\Omega \subset \mathbb{R}^{2}$. If its Hamiltonian $H(x, y)=$ $F(y)+G(x)$ has an isolated extremum at the origin $O$, then $O$ has a punctured neighbourhood covered with non-trivial cycles. We denote by $N_{O}$ the largest connected punctured neighbourhood of $O$ covered with non-trivial cycles. We define the period function $T: N_{O} \rightarrow \mathbb{R}$ of (1) as the function assigning to every point $(x, y) \in N_{O}$ the minimal period of the cycle passing through $(x, y)$. We say that the period function $T$ is increasing if, for every couple of cycles $\gamma_{1}, \gamma_{2}$, with $\gamma_{1}$ enclosed by $\gamma_{2}$, one has $T\left(\gamma_{1}\right) \leq T\left(\gamma_{2}\right)$. When $T$ is constant, we say that $O$ is isochronous. Let $\delta(s), s \in\left(\sigma_{*}, \sigma^{*}\right)$, be a curve of class $C^{1}$ meeting transversally the cycles of $N_{O}$. Assume that $\lim _{s \rightarrow \sigma_{*}^{+}} \delta(s)=O$. We can consider the function $T(s) \equiv T(\delta(s))$. Then $T$ is increasing if and only if $T(s)$ is a one-variable increasing function. Let $\gamma_{\bar{s}}$ be the unique cycle met by $\delta$ at the point $\delta(\bar{s})$. We say that $T$ has an extremum at $\gamma_{\bar{s}}$ if $T(s)$ has an extremum at $s=\bar{s}$. We say that $\gamma$ is a critical cycle if $\left[\frac{d}{d s} T(s)\right]_{s=\bar{s}}=0$. One can prove that this definition does not depend on the particular transversal curve $\delta$ chosen.

2000 Mathematics Subject Classification: 34C05, 34C07.

Key words and phrases: center, period function, convexity, critical orbit.

This work has been partially supported by the COFIN group "Equazioni differenziali ordinarie e applicazioni", and by the intergroup project "Dinamica anolonoma, perturbazioni e orbite periodiche". 
Studying the period function is essential in some stability, bifurcation, and boundary value problems related to Hamiltonian systems, or to systems reducible to Hamiltonian ones, as Lotka-Volterra systems. The period function's monotonicity for systems of type (1) was studied by several authors ([1], [6]-[9], [11]-[15]); here we do not mention papers devoted to isochronicity. In some cases the monotonicity was proved together with a convexity property related to $T$ ([14]), not implying T's convexity. Systems with a nonmonotone period function, hence with critical orbits, were studied in [2], [4], [5], [17]. In [12] it was proved that a system of type (1), with $F^{\prime}(y)=y$, can have at most a simple critical point for every central region, if $G(x)$ is a polynomial of degree four. In [3] it was proved that critical orbits of an analytic center do not accumulate on a compact set.

The monotonicity ensures that a typical boundary value problem, $x(0)=$ $x(T)$, has a unique solution for $T$ belonging to some interval. Similarly, when $F^{\prime}(y)=y$, that is, when the system takes the form

$$
x^{\prime}=y, \quad y^{\prime}=-G^{\prime}(x),
$$

the uniqueness of Neumann-like problems, $x^{\prime}(0)=x^{\prime}(T)$, may be reduced to the study of T's monotonicity, as in [1].

A different situation has to be taken into account when looking for multiple solutions of boundary value problems. If $x(0)=x(T)$ has more than a single solution, then $T(s)$ has different monotonicity properties in distinct intervals. Such intervals, corresponding to distinct subsets of $N_{O}$, are separated by values of $s$ where $T$ reaches a local extremum. The problem of counting the exact number of solutions to $x(0)=x(T)$ is related to the problem of counting such local extrema. The simplest way to estimate the number of such extrema is to study the convexity of $T(s)$, which ensures the uniqueness of the extremum. If $T(s)$ is convex, there exists an interval $\left(T_{1}, T_{2}\right)$ such that the BVP $x(0)=x(T)$ has exactly two solutions for $T \in\left(T_{1}, T_{2}\right)$.

In this paper we give sufficient conditions for the existence of a transversal curve $\delta(s)$ such that $T(\delta(s))$ is convex on some interval. The main tool applied is a theorem proved in [9], where $T$ was studied by means of a suitable auxiliary system,

$$
x^{\prime}=\frac{G(x)}{G^{\prime}(x)}, \quad y^{\prime}=\frac{F(y)}{F^{\prime}(y)} .
$$

Such a system is a normalizer of (1), that is, its local flow takes orbits of (1) into orbits of (1). If we denote by $V(x, y)$ the vector field of (1), and by $W(x, y)$ the vector field of (2), this is equivalent to saying that there exists a function $\mu: N_{O} \rightarrow \mathbb{R}$ such that

$$
[V, W]=\mu V .
$$


If $\delta(s)$ is a solution to $(2)$, then, as proved in [9],

$$
T^{\prime}(s)=\frac{d}{d s} T(\delta(s))=\int_{0}^{T(s)} \mu\left(\gamma_{s}(t)\right) d t .
$$

In the case of the couple of systems (1) and (2), one has

$$
\mu(x, y)=\left(\frac{G(x)}{G^{\prime}(x)}\right)^{\prime}+\left(\frac{F(y)}{F^{\prime}(y)}\right)^{\prime}-1 .
$$

Hence, proving the convexity of $T(s)$ reduces to proving that the integral in (3) has larger values on outer cycles. This can be done, on a suitable subset $A$ of $N_{O}$, by adapting a technique used to study the uniqueness of limit cycles in Liénard systems (see [10], [16], [18]).

In Theorem 1 we show that under suitable assumptions on the sign of some functions depending on $F, G$ and their derivatives up to the third order, $T^{\prime}(s)$ is increasing on $A$, hence $T(s)$ is convex on $A$. As a consequence, (1) has at most one critical orbit in $A$. Conditions for the existence and uniqueness of critical orbits are given for some classes of second order conservative ODE's. It is maybe worth noticing that the function $N(x)$ introduced in [1],

$$
N(x)=6 G(x) G^{\prime \prime 2}(x)-3 G^{\prime}(x)^{2} G^{\prime \prime}(x)-2 G(x) G^{\prime}(x) G^{\prime \prime \prime}(x),
$$

plays a role also in the study of convexity. On the other hand, we find an example of degenerate planar center with $T$ strictly decreasing at the origin, such that $N(x) \geq 0$ in a neighbourhood of $O$. This shows that Theorem A in [1] cannot be extended to degenerate centers.

2. Results. Let $G \in C^{3}(I, \mathbb{R}), F \in C^{3}(J, \mathbb{R}), I, J$ open intervals containing 0 , possibly unbounded. We consider the system (1), assuming $F$ and $G$ to have isolated minima at the origin. We do not assume the minima to be non-degenerate, because the results proved in [9] hold under the only assumption that $H(x, y)=G(x)+F(y)$ has a minimum at $O$. Also, we assume $x G^{\prime}(x)>0$ on $I \backslash\{0\}$, and $y F^{\prime}(y)>0$ on $J \backslash\{0\}$.

We say that (1) satisfies the conditions $(L)$ if there exist $\alpha \in C^{0}(I, \mathbb{R})$, $\beta \in C^{0}(J, \mathbb{R})$ and $a, b \in I, a \leq 0 \leq b, c, d \in J, c \leq 0 \leq d$, such that:

$\left(L_{1}\right) \quad \alpha(x)+\beta(y)=\left(\frac{G(x)}{G^{\prime}(x)}\right)^{\prime}+\left(\frac{F(y)}{F^{\prime}(y)}\right)^{\prime}-1$,

$\left(L_{2}\right) \quad \alpha(x) \geq 0$ for $x \notin[a, b], \alpha(x) F^{\prime \prime}(y) \leq 0$ for $x \in[a, b], y \notin[c, d]$;

$\left(L_{3}\right) \quad \beta(y) \geq 0$ for $y \notin[c, d], G^{\prime \prime}(x) \beta(y) \leq 0$ for $x \notin[a, b], y \in[c, d]$;

$\left(L_{4}\right) \quad\left(\frac{\alpha(x)}{G^{\prime}(x)}\right)^{\prime} \geq 0$ for $x \notin[a, b]$, 


$$
\left(L_{5}\right) \quad\left(\frac{\beta(y)}{F^{\prime}(y)}\right)^{\prime} \geq 0 \text { for } y \notin[c, d] .
$$

The above conditions are considered even in the case of intervals reducing to a single point, as it occurs when $a=0=b$.

We denote by $\mathcal{O}_{a b c d}^{e}$ the family of cycles contained in $N_{O}$ and enclosing the rectangle $[a, b] \times[c, d]$, and by $\mathcal{O}_{a b c d}^{i}$ the family of cycles contained in $N_{O} \cap[a, b] \times[c, d]$. In general, $N_{O} \neq \mathcal{O}_{a b c d}^{i} \cup \mathcal{O}_{a b c d}^{e}$. If $c=0=d, a<0<b$, we denote by $\mathcal{O}_{a b 00}^{e}$ the family of cycles meeting both the lines $x=a$ and $x=b$, and by $\mathcal{O}_{a b 00}^{i}$ the family of cycles contained in the strip $a<x<b$. Similarly for $a=0=b, c<0<d$.

Convexity is not assumed to be necessarily strict. Since there is oneto-one correspondence between the parameters $s$ and the orbits $\gamma_{s}$, we say equivalently that $T$ is (strictly) convex at $s$ or at $\gamma_{s}$. Similarly, we say that $T$ is (strictly) convex on $\mathcal{O}_{a b c d}^{e}$, or on $\mathcal{O}_{a b c d}^{i}$.

The main result of this paper is the following theorem.

Theorem 1. Assume that (1) satisfies the conditions $(L)$. Then the function $T$ is convex on $\mathcal{O}_{\text {abcd }}^{e}$.

Proof. It is sufficient to prove that $T^{\prime}(s)$ is increasing on $\mathcal{O}_{a b c d}^{e}$. By Lemma 7 in [9], the derivative of $T(s)$ is given by (3), where

$$
\mu(x, y)=\left(\frac{G(x)}{G^{\prime}(x)}\right)^{\prime}+\left(\frac{F(y)}{F^{\prime}(y)}\right)^{\prime}-1=\alpha(x)+\beta(y) .
$$

Consider two cycles, $\gamma_{s_{1}}, \gamma_{s_{2}}$, with $s_{1}<s_{2}$. The cycle $\gamma_{s_{1}}$ is contained in the bounded region having $\gamma_{s_{2}}$ as boundary. In order to prove that $T^{\prime}\left(s_{1}\right) \leq$ $T^{\prime}\left(s_{2}\right)$, we have to show that

$$
\int_{0}^{T\left(s_{1}\right)} \mu\left(\gamma_{s_{1}}(t)\right) d t \leq \int_{0}^{T\left(s_{2}\right)} \mu\left(\gamma_{s_{2}}(t)\right) d t .
$$

The orbits will be decomposed into arcs over which the integration will be performed with respect to $x$ or $y$.

Let us first compare the terms $\int_{0}^{T\left(s_{1}\right)} \alpha\left(\gamma_{s_{1}}(t)\right) d t$ and $\int_{0}^{T\left(s_{2}\right)} \alpha\left(\gamma_{s_{2}}(t)\right) d t$.

Since $\gamma_{1}$ encloses the rectangle $[a, b] \times[c, d]$, it meets the line $x=b$ at points $\left(b, c^{\prime}\right),\left(b, d^{\prime}\right)$, with $c^{\prime} \leq 0 \leq d^{\prime}$. Also, it meets the line $x=a$ at points $\left(a, c^{\prime \prime}\right),\left(a, d^{\prime \prime}\right)$, with $c^{\prime \prime} \leq 0 \leq d^{\prime \prime}$.

The curve $\gamma_{1}$ is the union of four arcs:

$$
\begin{array}{ll}
\gamma_{1}^{1} \subset\{a \leq x \leq b, y>0\}, & \gamma_{1}^{2} \subset\{x \geq b\}, \\
\gamma_{1}^{3} \subset\{a \leq x \leq b, y<0\}, & \gamma_{1}^{4} \subset\{x \leq a\} .
\end{array}
$$


The curve $\gamma_{2}$ is the union of eight arcs:

$$
\begin{array}{rlrl}
\gamma_{2}^{1} & \subset\{a \leq x \leq b, y>0\}, & \gamma_{2}^{2} \subset\left\{x \geq b, c^{\prime} \leq y \leq d^{\prime}\right\}, \\
\gamma_{2}^{3} \subset\{a \leq x \leq b, y<0\}, & \gamma_{2}^{4} \subset\left\{x \leq a, c^{\prime \prime} \leq y \leq d^{\prime \prime}\right\} ; \\
\gamma_{2}^{\mathrm{I}} \subset\left\{x \geq b, y \geq d^{\prime}\right\}, & \gamma_{2}^{\mathrm{II}} \subset\left\{x \geq b, y \leq c^{\prime}\right\}, \\
\gamma_{2}^{\mathrm{III}} \subset\left\{x \leq a, y \leq c^{\prime \prime}\right\}, & \gamma_{2}^{\mathrm{IV}} \subset\left\{x \leq a, y \geq d^{\prime \prime}\right\}
\end{array}
$$

(see Figure 1).

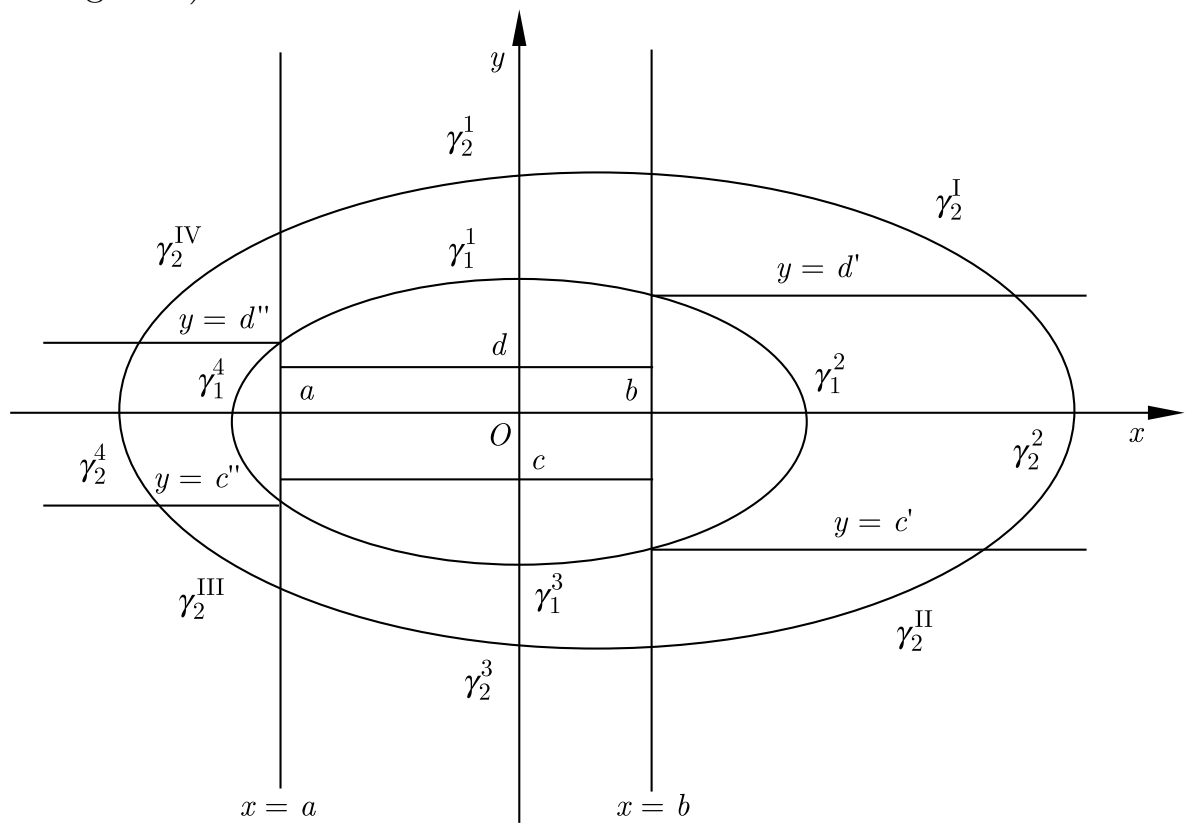

Since $\alpha \geq 0$ off $[a, b]$, one has

$$
\int_{\gamma_{2}^{\mathrm{I}}} \alpha \geq 0, \quad \int_{\gamma_{2}^{\mathrm{II}}} \alpha \geq 0, \quad \int_{\gamma_{2}^{\mathrm{III}}} \alpha \geq 0, \quad \int_{\gamma_{2}^{\mathrm{II}}} \alpha \geq 0 .
$$

In order to prove that $\int_{0}^{T\left(s_{1}\right)} \alpha\left(\gamma_{s_{1}}(t)\right) d t \leq \int_{0}^{T\left(s_{2}\right)} \alpha\left(\gamma_{s_{2}}(t)\right) d t$, it is sufficient to prove that

$$
\int_{\gamma_{1}^{j}} \alpha \leq \int_{\gamma_{2}^{j}} \alpha, \quad j=1, \ldots, 4 .
$$

We give the details only for the arcs $\gamma_{1}^{1}, \gamma_{1}^{2}, \gamma_{2}^{1}, \gamma_{2}^{2}$, since the other four arcs can be treated in a similar way. Since for $a \leq x \leq b$ one has $d x / d t=F^{\prime}(y)>0$, along $\gamma_{1}^{1}(t)$ one can express $t$ as a function of $x$ and integrate with respect to $x$. Writing $F(y)$ for $F(y(t(x)))$, one has

$$
\int_{\gamma_{1}^{1}} \alpha\left(\gamma_{s_{1}}(t)\right) d t=\left[\int_{a}^{b} \frac{\alpha(x) d x}{F^{\prime}(y)}\right]_{\gamma_{1}^{1}} .
$$


Since $\alpha(x) F^{\prime \prime}(y) \leq 0$ for $x \in[a, b], y \notin[c, d]$, one has

$$
\frac{\partial}{\partial y} \frac{\alpha(x)}{F^{\prime}(y)}=-\frac{\alpha(x) F^{\prime \prime}(y)}{F^{\prime}(y)^{2}} \geq 0,
$$

so that $\alpha(x) / F^{\prime}(y)$ is an increasing function of $y$. As $\gamma_{2}$ is external with respect to $\gamma_{1}$, it follows that

$$
\int_{\gamma_{1}^{1}} \alpha\left(\gamma_{s_{1}}(t)\right) d t=\left[\int_{a}^{b} \frac{\alpha(x) d x}{F^{\prime}(y)}\right]_{\gamma_{1}^{1}} \leq\left[\int_{a}^{b} \frac{\alpha(x) d x}{F^{\prime}(y)}\right]_{\gamma_{2}^{1}}=\int_{\gamma_{2}^{1}} \alpha\left(\gamma_{s_{2}}(t)\right) d t .
$$

Now consider the arcs $\gamma_{1}^{2}, \gamma_{2}^{2}$, along which one has $d y / d t=-G^{\prime}(x)<0$, so that one can express $t$ as a function of $y$, and integrate with respect to $y$,

$$
\int_{\gamma_{1}^{2}} \alpha\left(\gamma_{s_{1}}(t)\right) d t=\left[\int_{d^{\prime}}^{c^{\prime}} \frac{\alpha(x) d y}{-G^{\prime}(x)}\right]_{\gamma_{1}^{2}}=\left[\int_{c^{\prime}}^{d^{\prime}} \frac{\alpha(x) d y}{G^{\prime}(x)}\right]_{\gamma_{1}^{2}} .
$$

By $\left(L_{4}\right)$, one has

$$
\frac{\partial}{\partial x}\left(\frac{\alpha(x)}{G^{\prime}(x)}\right) \geq 0
$$

hence $\alpha(x) / G^{\prime}(x)$ is an increasing function, and as above

$$
\int_{\gamma_{1}^{2}} \alpha\left(\gamma_{s_{1}}(t)\right) d t=\left[\int_{d^{\prime}}^{c^{\prime}} \frac{\alpha(x) d y}{-G^{\prime}(x)}\right]_{\gamma_{1}^{2}} \leq\left[\left[\int_{d^{\prime}}^{c^{\prime}} \frac{\alpha(x) d y}{-G^{\prime}(x)}\right]_{\gamma_{1}^{2}}\right]_{\gamma_{2}^{2}}=\int_{\gamma_{2}^{2}} \alpha\left(\gamma_{s_{2}}(t)\right) d t .
$$

The same argument works as well for the $\operatorname{arcs} \gamma_{1}^{3}, \gamma_{1}^{4}, \gamma_{2}^{3}, \gamma_{2}^{4}$. Summing up, one has

$$
\int_{0}^{T\left(s_{1}\right)} \alpha\left(\gamma_{s_{1}}(t)\right) d t \leq \int_{0}^{T\left(s_{2}\right)} \alpha\left(\gamma_{s_{2}}(t)\right) d t
$$

Now consider the integrals involving $\beta$. We can work as we did for $\alpha$, with the lines $y=c, y=d$ playing the role of $x=a, x=b$. Computations are similar, and lead to a similar conclusion,

$$
\int_{0}^{T\left(s_{1}\right)} \beta\left(\gamma_{s_{1}}(t)\right) d t \leq \int_{0}^{T\left(s_{2}\right)} \beta\left(\gamma_{s_{2}}(t)\right) d t
$$

The term -1 appearing in $\mu$ can be absorbed in different ways by $\alpha$ and $\beta$. In general, for a given $\kappa \in \mathbb{R}$, we may write

$$
\mu(x, y)=\left[\left(\frac{G(x)}{G^{\prime}(x)}\right)^{\prime}+\kappa\right]+\left[\left(\frac{F(y)}{F^{\prime}(y)}\right)^{\prime}-1-\kappa\right]=\alpha(x)+\beta(y) .
$$

Denote by $\left(-L_{j}\right), j=2, \ldots, 5$, the conditions obtained from $\left(L_{j}\right), j=$ $2, \ldots, 5$, by reversing the inequalities. We have the following analogue of Theorem 1 for the concavity of the period function. 
Theorem 2. Assume that (1) satisfies the conditions $\left(L_{1}\right),\left(-L_{j}\right), j=$ $2, \ldots, 5$. Then the function $T$ is concave on $\mathcal{O}_{a b c d}^{e}$.

Proof. As in Theorem 1, reversing the integral inequalities.

The next four corollaries are concerned with the strict convexity on $\mathcal{O}_{a b c d}^{e}$. This property implies the uniqueness of critical orbits on $\mathcal{O}_{a b c d}^{e}$, if they exist.

Corollary 1. Assume that the hypotheses of Theorem 1 hold. Let the cycle $\bar{\gamma}$ pass through a point $(\bar{x}, \bar{y})$ such that at least one of the inequalities in $\left(L_{j}\right), j=2, \ldots, 5$, is strict. Then $T$ is strictly convex in a neighbourhood of $\bar{\gamma}$.

Proof. At least one of the integral inequalities of the proof of Theorem 1 is strict at $(\bar{x}, \bar{y})$. By continuity, this holds in a neighbourhood of $(\bar{x}, \bar{y})$, hence $T^{\prime}(s)$ is strictly increasing in a neighbourhood of $\bar{\gamma}$.

For instance, if there exists $\bar{x}>b$ such that $\alpha(\bar{x})>0$, then $T$ is strictly convex at every orbit intersecting the line $x=\bar{x}$. As a consequence, one has at most one critical orbit intersecting the line $x=\bar{x}$. A similar statement can be proved about strict concavity.

COROLlary 2. Under the hypotheses of Theorem 1, assume that one of the following holds:

(i) there exist $x_{n}>b$ with $\lim _{n \rightarrow \infty} x_{n}=b$ such that $\alpha\left(x_{n}\right)>0\left(x_{n}<a\right.$ with $\lim _{n \rightarrow \infty} x_{n}=$ a such that $\alpha\left(x_{n}\right)>0$ );

(ii) there exist $y_{n}>d$ with $\lim _{n \rightarrow \infty} y_{n}=d$ such that $\beta\left(y_{n}\right)>0\left(y_{n}<c\right.$ with $\lim _{n \rightarrow \infty} y_{n}=c$ such that $\left.\beta\left(y_{n}\right)>0\right)$.

Then the function $T$ is strictly convex on $\mathcal{O}_{a b c d}^{e}$.

Proof. This is an immediate consequence of Corollary 1 , since every cycle in $\mathcal{O}_{a b c d}^{e}$ has to meet at least one of the lines $x=x_{n}\left(y=y_{n}\right)$.

COROLlary 3. Under the hypotheses of Theorem 1, assume that one of the following holds:

(i) there exists $\bar{x} \in[a, b]$ such that $\alpha(\bar{x})<0$ and $F^{\prime \prime}(y)>0$ for $y>d$ $\left(F^{\prime \prime}(y)>0\right.$ for $\left.y<c\right)$;

(ii) there exists $\bar{y} \in[c, d]$ such that $\beta(\bar{y})<0$ and $G^{\prime \prime}(x)>0$ for $x>b$ $\left(G^{\prime \prime}(x)>0\right.$ for $\left.x<a\right)$.

Then the function $T$ is strictly convex on $\mathcal{O}_{a b c d}^{e}$.

Proof. (i) is an immediate consequence of Corollary 1, since every cycle in $\mathcal{O}_{a b c d}^{e}$ has to meet the half-line $x=\bar{x}, y>d(x=\bar{x}, y<c)$. Item (ii) can be proved similarly.

Strict convexity (concavity) can also be proved for analytic systems. We recall that monotonicity is not assumed to be strict, so that a constant period function is monotone. 
Corollary 4. Assume that the hypotheses of Theorem 1 hold. If $F$ and $G$ are analytic functions, and $T$ is not monotone on $\mathcal{O}_{\text {abcd }}^{e}$, then $T$ is strictly convex on $\mathcal{O}_{a b c d}^{e}$.

Proof. $T(s)=T(\delta(s))$ is an analytic function. By Theorem $1, T$ is convex on $\mathcal{O}_{a b c d}^{e}$, hence $T^{\prime \prime}(s) \geq 0$. Moreover, $T^{\prime \prime}(s)$ is not identically zero: otherwise there would exist $\kappa_{1}, \kappa_{2} \in \mathbb{R}$ such that $T(s)=\kappa_{1} s+\kappa_{2}$, which would imply monotonicity. By analyticity, the zeroes of $T^{\prime \prime}(s)$ are isolated, so $T^{\prime}(s)$ is strictly increasing, which gives the strict convexity of $T$.

EXAmple 1. Setting

$$
G(x)=\frac{|x|^{11 / 2}}{x^{4}+1}
$$

consider the Hamiltonian

$$
H(x, y)=G(x)+G(y)=\frac{|x|^{11 / 2}}{x^{4}+1}+\frac{|y|^{11 / 2}}{y^{4}+1} .
$$

Since $G(x)$ is an even function, one may consider only its derivatives for $x>0$, which simplifies the computations. One has, for $x>0$,

$$
G^{\prime}(x)=x^{9 / 2} \frac{3 x^{4}+11}{2\left(x^{4}+1\right)^{2}}, \quad G^{\prime \prime}(x)=x^{7 / 2} \frac{3 x^{8}-26 x^{4}+99}{4\left(x^{4}+1\right)^{3}},
$$

hence the origin is a global center, with convex Hamiltonian. Moreover, setting

$$
\alpha(x)=\frac{3 x^{8}+118 x^{4}-77}{2\left(3 x^{4}+11\right)^{2}},
$$

one has

$$
\mu(x, y)=\alpha(x)+\alpha(y)=\frac{3 x^{8}+118 x^{4}-77}{2\left(3 x^{4}+11\right)^{2}}+\frac{3 y^{8}+118 y^{4}-77}{2\left(3 y^{4}+11\right)^{2}} .
$$

The function $\alpha(x)$ vanishes for $x_{0}=\left(-\frac{59}{3}+\frac{8}{3} \sqrt{58}\right)^{1 / 4}$, which is approximately 0.9. One has $\alpha(x)<0$ for $x \in\left(-x_{0}, x_{0}\right)$, and $\alpha(x)>0$ for $x \notin$ $\left[-x_{0}, x_{0}\right]$. The period function of the Hamiltonian system is decreasing on the orbits contained in the square $Q=\left[-x_{0}, x_{0}\right] \times\left[-x_{0}, x_{0}\right]$, and is strictly convex on the orbits enclosing $Q$.

The next corollary is concerned with conservative second order differential equations,

$$
x^{\prime \prime}+G^{\prime}(x)=0 .
$$

As in [1], we set

$$
N(x)=6 G(x) G^{\prime \prime 2}(x)-3 G^{\prime}(x)^{2} G^{\prime \prime}(x)-2 G(x) G^{\prime}(x) G^{\prime \prime \prime}(x) .
$$

In what follows, we choose $c=0=d$. 
Corollary 5. Let $G \in C^{3}(I, \mathbb{R})$ with $x G^{\prime}(x)>0$ for $x \neq 0$. If there exist $a, b \in I, a \leq 0 \leq b$, such that

(i) $G^{\prime}(x)^{2}-2 G(x) G^{\prime \prime}(x) \leq 0$ for $x \in[a, b]$, and $G^{\prime}(x)^{2}-2 G(x) G^{\prime \prime}(x) \geq 0$ for $x \notin[a, b]$,

(ii) $N(x) \geq 0$ for $x \notin[a, b]$,

then the period function $T(s)$ is convex on $\mathcal{O}_{a b 00}^{e}$.

Reversing the above inequalities implies the concavity of $T(s)$ on $\mathcal{O}_{a b 00}^{e}$.

Proof. The equation (4) is a special case of (1), with $F(y)=y^{2} / 2$, $c=0=d, \beta(y)=0$. Then $\alpha=\left(G^{2}-2 G G^{\prime \prime}\right) / 2 G^{2}$, and

$$
\left(\frac{\alpha}{G^{\prime}}\right)^{\prime}=\frac{6 G G^{\prime \prime 2}-3 G^{\prime 2} G^{\prime \prime}-2 G G^{\prime} G^{\prime \prime \prime}}{2 G^{\prime 4}}=\frac{N}{2 G^{\prime 4}} .
$$

Conditions (i)-(ii) ensure that the hypotheses of Theorem 1 hold.

A simple additional condition allows us to prove the uniqueness of critical orbits of (4) on all of $N_{O}$. In the situation considered in the next corollary, one has $N_{O}=\mathcal{O}_{a b 00}^{i} \cup \mathcal{O}_{a b 00}^{e}$.

Corollary 6. Suppose that (4) is a non-linear equation. Under the hypotheses of Corollary 5, assume additionally that $G(a)=G(b)$. If the hypotheses of one of the corollaries 2 or 4 hold, then (4) has at most one critical orbit in $N_{O}$, contained in the set $G(x)+y^{2} / 2>G(a)$.

Proof. The cycles are contained in level sets of the first integral $G(x)+$ $y^{2} / 2$. If $G(a)=G(b)$, then there exists a cycle $\gamma_{a b}$ passing through $(a, 0)$ and $(b, 0)$. All the other cycles either meet both the lines $x=a$ and $x=b$, or are contained in the strip $a<x<b$, hence $N_{O}=\mathcal{O}_{a b 00}^{i} \cup \mathcal{O}_{a b 00}^{e}$. One has $T^{\prime}(s) \leq 0$ for every cycle $\gamma_{s} \in \mathcal{O}_{a b 00}^{i}$, because $\alpha(x) \leq 0$ on $[a, b]$. We claim that actually $T^{\prime}(s)<0$ on $\mathcal{O}_{a b 00}^{i}$. In fact, assume that $\alpha \equiv 0$ on $[a, b]$. Then $G^{2}-2 G G^{\prime \prime} \equiv 0$ on $[a, b]$, so that, on the interval $(0, b)$, where both $G$ and $G^{\prime}$ are positive, one has

$$
\frac{G^{\prime}}{G}=2 \frac{G^{\prime \prime}}{G^{\prime}}
$$

Integrating gives $\ln G=2 \ln G^{\prime}+\kappa_{0}, \kappa_{0} \in \mathbb{R}$, hence $G=\kappa_{1} G^{2}, \kappa_{1}>0$. Integrating the equation $G=\kappa_{1} G^{2}$ gives $G(x)=\left(\kappa_{2} x+\kappa_{3}\right)^{2}$. Since $G(x)$ vanishes at 0 , one has $\kappa_{3}=0$, so that $G(x)=\left(\kappa_{2} x\right)^{2}$, contradicting the non-linearity of (4). This proves that $\alpha(x)$ vanishes identically on no interval $\left[0, b_{1}\right) \subset[0, b)$. As a consequence, $T^{\prime}$ is strictly negative on $\mathcal{O}_{a b 00}^{i}$. In particular, $T^{\prime}$ is strictly negative on the orbit $\gamma_{a b}$, and, by continuity, on a neighbourhood of $\gamma_{a b}$. Hence a critical orbit cannot be contained in the sublevel set $G(x)+y^{2} / 2 \leq G(a)$, but, if it exists, it has to belong to $\mathcal{O}_{a b 00}^{e}$, where $T$ is strictly convex, by Corollary 2 or 4 . This gives the uniqueness. 
EXAmple 2. The potential $G(x)=x^{2}+x^{4}-x^{6}$ generates the system

$$
x^{\prime}=y, \quad y^{\prime}=-2 x-4 x^{3}+6 x^{5} .
$$

We take $I=[-1,1]$ and $J=\mathbb{R}$. The system (5) has a center at the origin, with central region contained in the rectangle $[-1,1] \times[-\sqrt{2}, \sqrt{2}]$.

One has

$$
\begin{aligned}
& G^{2}-2 G G^{\prime \prime}=-4 x^{4}\left(3-8 x^{2}-9 x^{4}+6 x^{6}\right), \\
& N=-24 x^{4}\left(1-18 x^{2}+34 x^{4}-52 x^{6}-59 x^{8}+30 x^{10}\right) .
\end{aligned}
$$

Applying the Sturm procedure, one can show that in the interval $[-1,1]$, $G^{\prime 2}-2 G G^{\prime \prime}$ has exactly two zeroes $-x_{1}<0<x_{1}$, as does $N$, which vanishes at $-x_{2}<0<x_{2}$. One has $-x_{1}<-x_{2}<0<x_{2}<x_{1}$, so that if we take $a=-x_{1}, b=x_{1}$, the system (5) satisfies all the hypotheses of Corollary 6 . Its period function is strictly decreasing in a neighbourhood of the origin, it is strictly convex on $\mathcal{O}_{-x_{1} x_{1} 00}$, it tends to $+\infty$ approaching the boundary $\partial N_{O}$, and there exists exactly one critical orbit. A numerical approximation shows that $x_{1}$ is approximately 0.544 , while $x_{2}$ is approximately 0.249 .

EXAmple 3 . The potential $G(x)=\frac{x^{4}}{x^{4}+1}$ generates the system

$$
x^{\prime}=y, \quad y^{\prime}=-\frac{4 x^{3}}{\left(x^{4}+1\right)^{2}} .
$$

We take $I=\mathbb{R}, J=(-\sqrt{2}, \sqrt{2})$. The system (6) has a center at the origin, with central region contained in the strip $I \times J$. One has

$$
G^{2}-2 G G^{\prime \prime}=\frac{8 x^{6}\left(5 x^{4}-1\right)}{\left(x^{4}+1\right)^{4}} .
$$

The right hand side is negative for $x \in\left(-1 / 5^{1 / 4}, 1 / 5^{1 / 4}\right)$, and positive for $x \notin\left[-1 / 5^{1 / 4}, 1 / 5^{1 / 4}\right]$. Moreover, one has

$$
N=96 x^{8}\left(15 x^{8}+1\right) /\left(x^{4}+1\right)^{7},
$$

which is positive for $x \neq 0$. Also in this example $T^{\prime}(s)<0$ on the cycles contained in the strip $x \in\left[-1 / 5^{1 / 4}, 1 / 5^{1 / 4}\right]$, and $T$ is strictly convex on the cycles meeting both the lines $x= \pm 1 / 5^{1 / 4}$. As a consequence, the system (6) has exactly one critical cycle, meeting both the lines $x= \pm 1 / 5^{1 / 4}$.

REMARK 1. The above example shows that Theorem A in [1] cannot be extended to non-degenerate centers. In fact, the function $N(x)$ is positive everywhere but at 0 while $T$ is strictly decreasing in a neighbourhood of the origin. The proof of Theorem A in [1] does not apply because the center of (6) is degenerate, and the change of variables on which the proof is based cannot be defined. 


\section{References}

[1] C. Chicone, The monotonicity of the period function for planar Hamiltonian vector fields, J. Differential Equations 69 (1987), 310-321.

[2] C. Chicone and F. Dumortier, A quadratic system with a nonmonotonic period function, Proc. Amer. Math. Soc. 102 (1988), 706-710.

[3] - - - Finiteness for critical periods of planar analytic vector fields, Nonlinear Anal. 20 (1993), 315-335.

[4] C. Chicone and M. Jacobs, Bifurcation of critical periods for plane vector fields, Proc. Amer. Math. Soc. 102 (1988), 706-710.

[5] S. N. Chow and J. A. Sanders, On the number of critical points of the period, J. Differential Equations 64 (1986), 51-66.

[6] S. N. Chow and D. Wang, On the monotonicity of the period function of some second order equations, Casopis Pěst. Mat. 111 (1986), 14-25.

[7] A. Cima, A. Gasull and F. Mañosas, Period function for a class of Hamiltonian systems, J. Differential Equations 168 (2000), 180-199.

[8] W. A. Coppel and L. Gavrilov, The period function of a Hamiltonian quadratic system, Differential Integral Equations 6 (1993), 1357-1365.

[9] E. Freire, A. Gasull and A. Guillamon, First derivative of the period function with applications, J. Differential Equations 204 (2004), 139-162.

[10] A. Gasull and A. Guillamon, Non-existence, uniqueness of limit cycles and center problem in a system that includes predator-prey systems and generalized Liénard equations, Differential Equations Dynam. Systems 3 (1995), 345-366.

[11] A. Gasull, A. Guillamon and J. Villadelprat, The period function for second-order quadratic ODEs is monotone, preprint, Univ. Aut. de Barcelona, 2003.

[12] L. Gavrilov, Remark on the number of critical points of the period, J. Differential Equations 101 (1993), 58-65.

[13] F. Rothe, The periods of the Lotka-Volterra systems, J. Reine Angew. Math. 355 (1985), 129-138.

[14] - Remarks on the periods of planar Hamiltonian systems, SIAM J. Math. Anal. 24 (1993), 129-154.

[15] R. Schaaf, A class of Hamiltonian systems with increasing periods, J. Reine Angew. Math. 363 (1985), 96-109.

[16] G. Villari, Some remarks on the uniqueness of the periodic solutions for Liénard's equation, Boll. Un. Mat. Ital. C (6) 4 (1985), 173-182.

[17] D. Wang, The critical points of the period function of $x^{\prime \prime}-x^{2}(x-\alpha)(x-1)(0 \leq$ $\alpha<1$ ), Nonlinear Anal. 11 (1987), 1029-1050.

[18] D. Xiao and Z. Zhang, On the uniqueness and nonexistence of limit cycles for predator-prey systems, Nonlinearity 16 (2003), 1185-1201.

Dipartimento di Matematica

Università di Trento

I-38050 Povo (TN), Italy

E-mail: marco.sabatini@unitn.it 